\title{
Vivências comunitárias durante as excursões didáticas no curso de Odontologia da UFPE - uma experiência extramuros
}

Márcia Maria Vendiciano Barbosa Vasconcelos*; José Thadeu Pinheiro**; Arnaldo de França Caldas Júnior***; André Cavalcante da Silva Barbosa****; Amanda Priscilla Santana da Silva*****; Elizabeth Louisy Marques Soares da Silva*****

\section{RESUMO}

O objetivo deste trabalho foi avaliar a percepção dos alunos envolvidos no projeto de Excursão Didática a respeito da integralidade da atenção à saúde e da integralização ensino, trabalho e práticas sociais. Trata-se de um estudo observacional do tipo analítico. A amostra foi composta de 109 alunos que participaram das excursões e que estavam cursando entre o $7^{\circ}$ e o $10^{\circ}$ período do curso de Odontologia da Universidade Federal de Pernambuco (UFPE). As atividades das excursões foram desenvolvidas nas cidades do sertão pernambucano. O questionário aplicado para coletar as informações dos participantes foi composto por quatro partes: Dados pessoais (nome, idade, sexo e período do curso);
Professora Associada do Departamento de Clínica e Odontologia Preventiva, UFPE

** Professor Titular do Departamento de Prótese e Cirurgia Buco-Facial, UFPE

*** Professor Adjunto do Departamento de Clínica e Odontologia Preventiva, UFPE

*** Doutorando em Odontologia, Programa de Pósgraduação em Odontologia, UFPE

Cirurgiã-dentista
Infraestrutura necessária para a excursão (transporte, recurso financeiro e duração da atividade); Abordagem clínica (acolhimento, relacionamento dos profissionais com os pacientes e famílias, biossegurança e domínio das atividades clínicas); e Resultado final da excursão. Quase a totalidade da amostra dos estudantes entendeu como adequada a metodologia utilizada durante todo o transcurso da atividade. As atividades extramuros desenvolvidas nas comunidades cumpriram seu principal objetivo, que foi proporcionar aos alunos uma visão integral da realidade social e sanitária encontrada nas comunidades não assistidas pelos serviços básicos de saúde.

Descritores: Ensino. Odontologia Comunitária. Educação em Odontologia. 


\section{INTRODUÇÃO}

As pessoas estão habituadas a associar boa saúde com assistência à saúde, isto é, com a possibilidade que elas têm de se consultar com médicos e dentistas, realizar exames ou se internar em hospitais ${ }^{1}$. Esse modelo de prática vem sendo exercido há mais de um século e essa prática médica e odontológica não conseguiu obter melhores níveis de saúde para a humanidade ${ }^{1}$. Sabe-se que, em geral, hoje se vive mais e melhor do que há 100 anos. Mas isso se deu não como decorrência do modelo de intervenção médica hegemônica e sim devido às melhores condições sociais de existência, possíveis nos dias atuais ${ }^{1}$.

A possibilidade de uma pessoa alcançar o "bem-estar físico, mental e social" vai depender do grupo social ao qual ela pertença. Vai depender ainda da ocupação principal da pessoa, quanto ela ganha, nível de escolaridade, da possibilidade de acesso maior a informações, das participações maior ou menor nas esferas de decisão política, do consumo (água potável, alimentos, moradia, transporte, vestuário, lazer) e de acesso a serviços de saúde ${ }^{2}$. Em relação a estes aspectos de melhorias das condições de vida, as oportunidades de promoção de saúde são mais legítimas ${ }^{1}$.

O que caracteriza a promoção da saúde, na atualidade, é a constatação de que a saúde tem uma determinação social, portanto está relacionada com a totalidade da vida. Pode-se afirmar que o enfoque da Promoção da Saúde é mais amplo e abrangente e consequente a uma mudança de visão do que é saúde ${ }^{3}$. A Promoção da Saúde é considerada um instrumental conceitual, político e metodológico em torno do processo saúdedoença, que visa analisar e atuar sobre as condições sociais que são críticas para melhorar as condições de saúde e de qualidade de vida ${ }^{3}$.

De acordo com a carta de Ottawa, a Promoção da Saúde é o processo que visa aumentar a capacidade dos indivíduos e das comunidades para controlarem a sua saúde, no sentido de melhorá-la. Para atingir um estado de completo bem-estar físico, mental e social, o indivíduo ou o grupo devem estar aptos a identificar e realizar as suas aspirações, a satisfazer as suas necessidades e a modificar ou adaptar-se ao meio 4 .

Embasados nesses conhecimentos, os profissionais de saúde devem entender o indivíduo como um ser humano integral, submetidos às mais diferentes situações de vida e trabalho que o levam a adoecer e a morrer ${ }^{5}$. Os indivíduos não devem ser vistos como amontoados de partes (coração, fígado, pulmões) que estão "soltos no mundo". São seres sociais, cidadãos que biologicamente, psicologicamente e socialmente estão sujeitos a riscos. No Sistema Único de Saúde (SUS), o conceito de integralidade tem sido trabalhado sob diferentes enfoques, o qual o caracteriza não apenas como uma diretriz, mas como uma imagem-objetivo para se fazer justiça social, que deve ser pautada na configuração das políticas, do sistema de saúde e na instituição das práticas de saúde. A atenção deve se pautar na sua saúde e não somente na assistência às doenças, devendo coexistir ações que busquem minimizar as causas das doenças, os riscos de diferentes ordens e tratar os danos. Portanto, deve integrar ações de promoção, proteção, recuperação e manutenção ${ }^{6}$.

Sendo assim, as atividades de extensão (AE) universitária foram criadas com a finalidade de reorientar os projetos pedagógicos na formação do profissional da saúde, o qual deve ser sensível às necessidades sociais e ter competência para 
gerar mudanças no quadro epidemiológico das doenças, a partir da concepção de integralidade em saúde e consequente melhoria da qualidade de vida da população. Essas atividades foram concebidas com o propósito de contribuir para formação de um profissional sensível às necessidades de saúde da população, buscando a integração multidisciplinar, entre ações de natureza preventiva e curativa, teoria e prática, e entre ensino e serviço ${ }^{7}$. Vem se fortalecendo a compreensão da extensão universitária como processo educativo, cultural e científico, que articula o ensino e a pesquisa viabilizando encontros e diálogos entre alunos, professores e com a sociedade indicando a possibilidade de produção de novos conhecimentos, de caráter emancipador constituídos a partir do movimento de troca e construção entre os saberes científico e popular. Assim, entendese que a extensão possui algumas características potencializadoras de mudanças ${ }^{8}$.

No retorno à universidade, docentes $\mathrm{e}$ discentes trarão um aprendizado que, submetido à reflexão teórica, será acrescido de conteúdos para sua construção. Esse fluxo, que estabelece a troca de saberes sistematizados, acadêmico e popular, terá como consequências a produção do conhecimento resultante do confronto com a realidade brasileira e regional, a democratização do conhecimento acadêmico e a participação efetiva da comunidade na atuação da universidade. Além de instrumentalizadora deste processo dialético de teoria/prática, a extensão é um trabalho interdisciplinar que favorece a visão integrada do social ${ }^{9}$.

A exploração de resultados positivos alcançados em projetos de extensão é importante, pois gera espaço para outras áreas do conhecimento com o envolvimento de outros departamentos. Também fortalece o processo da interdisciplinaridade, bem como o entrosamento com outras atividades comunitárias e sociais, graças ao contato direto com o meio, o que permite a retroalimentação obtida nas palestras dialogadas, em reuniões com a comunidade e trabalhando a própria realidade que resulta das necessidades manifestadas espontaneamente pela cidadania local, propiciando um processo de transformação, mesmo em longo prazo, da educação em saúde bucal tão necessária e urgente ${ }^{10}$.

As Excursões Didáticas são atividades complementares e fundamentais na formação acadêmica dos alunos. São desenvolvidas fora das salas de aula e fazem parte do conteúdo programático de diversas disciplinas obrigatórias e eletivas de diferentes cursos de graduação e pós-graduação. Essas atividades de campo são realizadas fora do campus universitário durante os semestres letivos, de acordo com o calendário acadêmico, envolvendo os estudantes regularmente matriculados, técnicos e professores responsáveis pelas disciplinas, na coleta de material e visitas de natureza científica e cultural em áreas geográficas de acesso acidentado, sítios históricos em áreas urbanas e rurais ${ }^{11}$.

A prática de campo propicia espaços e condições aos acadêmicos além de intensa relação entre a teoria e a prática, desperta no aluno uma consciência global e crítica a respeito das condições de saúde bucal da população e, das ações de saúde coletiva, bem como, promove ações de promoção de saúde, integrando a Universidade a realidade da comunidade. Objetiva-se neste trabalho avaliar como os atores sociais envolvidos no projeto de Excursões Didáticas percebem a integralidade da atenção à saúde e a vinculação entre educação, trabalho e práticas sociais. 


\section{METODOLOGIA}

Tratou-se de um estudo observacional do tipo analítico, o qual teve como população alvo os alunos que participaram das Excursões Didáticas do curso de Odontologia nos semestres 2012/2, 2013/1 e 2014/1. As atividades das excursões foram desenvolvidas nas cidades do sertão pernambucano, Arcoverde e Manari (outubro 2012), Ibimirim (março 2013) e Tuparetama (2014).

Foram aplicados 117 questionários aos alunos que participaram das excursões e que estavam cursando entre o $7^{\circ}$ e o $10^{\circ}$ período do curso de Odontologia da Universidade Federal de Pernambuco (UFPE). O referido questionário foi validado por meio de sua aplicação em dois alunos de cada período, os quais não fizeram parte da amostra final, que resultou em 109 indivíduos.

O questionário, aplicado para coletar as informações dos participantes da excursão didática foi composto de quatro partes: Dados pessoais (nome, idade, sexo e período do curso de graduação); Infraestrutura necessária para a excursão (meio de transporte, recurso financeiro e duração da atividade); Abordagem clínica (acolhimento, relacionamento dos profissionais com os pacientes e famílias, biossegurança e domínio das atividades clínicas) e Resultado final da excursão.

O projeto foi aprovado pelo Comitê de Ética da UFPE (CAAE: 20553113.7. 0000.5208) e no seu desenvolvimento foi observada a Resolução CNS 196/96, sendo que todos os indivíduos entrevistados foram convidados a participar e assinaram o Termo de Consentimento Livre e Esclarecido.

\section{RESULTADOS}

Foram entrevistados 109 alunos, sendo $25,69 \%$ do sexo masculino e $74,31 \%$ do sexo feminino. Com relação ao período do curso, $4,58 \%$ cursavam o $7^{\circ}$ período, $39.45 \%$ estavam no $8^{\circ}, 34,86 \%$ no $9^{\circ}$ e $21,10 \%$ no $10^{\circ}$. Dos pesquisados, $46,78 \%$ realizaram apenas uma excursão didática até o momento da entrevista, $48,62 \%$ excursionaram duas vezes e 3,67\% participaram da atividade em três oportunidades (gráfico 1).

O recurso financeiro ( $\mathrm{R} \$ 135,00)$ recebido pelos alunos para custear despesas de hospedagem foi considerado insatisfatório por $68,80 \%$, por outro lado, 70,64\% consideraram satisfatório o número de dias designados para a realização da atividade (04 dias). Quanto ao meio de transporte empregado na viagem (ônibus), 99,08\% referiram como adequado (gráfico 2).

Realizando uma análise descritiva das questões relacionadas ao atendimento odontológico proposto para a excursão, observou-se que $96,33 \%$ dos estudantes entenderam como adequado o acolhimento aos pacientes durante todo o transcurso da atividade (gráfico 3).

No quesito biossegurança, 85,32\% dos alunos consideraram adequadas as medidas utilizadas. A quantidade de instrumental e de material de consumo disponível para o atendimento foi avaliada, nesse quesito $67,89 \%$ entenderam como suficiente o número de instrumentais, enquanto que $78,90 \%$ consideraram a quantidade de material de consumo suficiente para a realização dos atendimentos. A logística empregada na esterilização dos instrumentais foi avaliada como adequada por $90,82 \%$ dos alunos entrevistados (gráfico 3).

Dos usuários menores de 18 anos, $41 \%$ estavam acompanhados pelos pais/ responsáveis, enquanto $18 \%$ se apresentaram desacompanhados, visto que estavam em horário escolar, porém com autorização prévia 
dos pais para receber o tratamento proposto (gráfico 4).

Os alunos participantes da excursão também foram questionados sobre sua percepção a respeito do modelo de atendimento odontológico utilizado, aproximadamente $\quad 35 \% \quad$ consideraram adequados, $31 \%$ humanizado e adequado de acordo com as circunstâncias, $32 \%$ humanizado (gráfico 5).

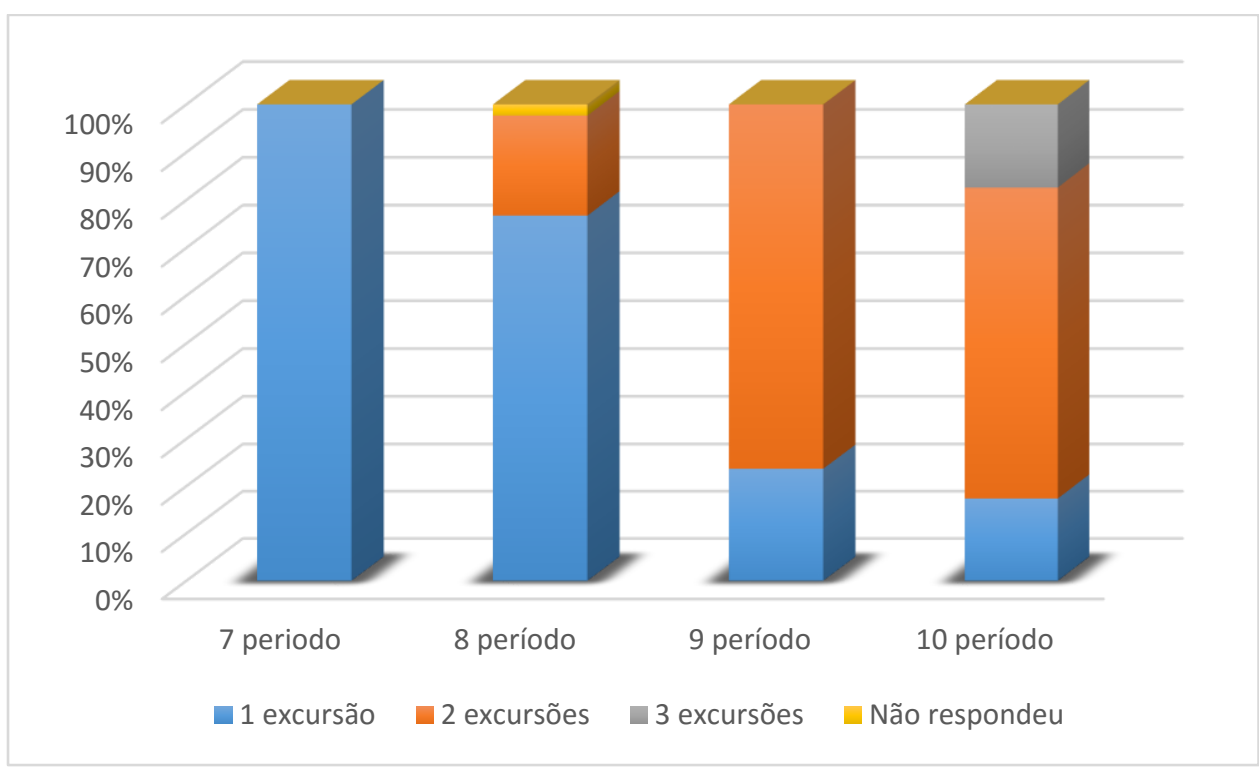

Gráfico 1. Número de excursões nas quais os estudantes participaram.

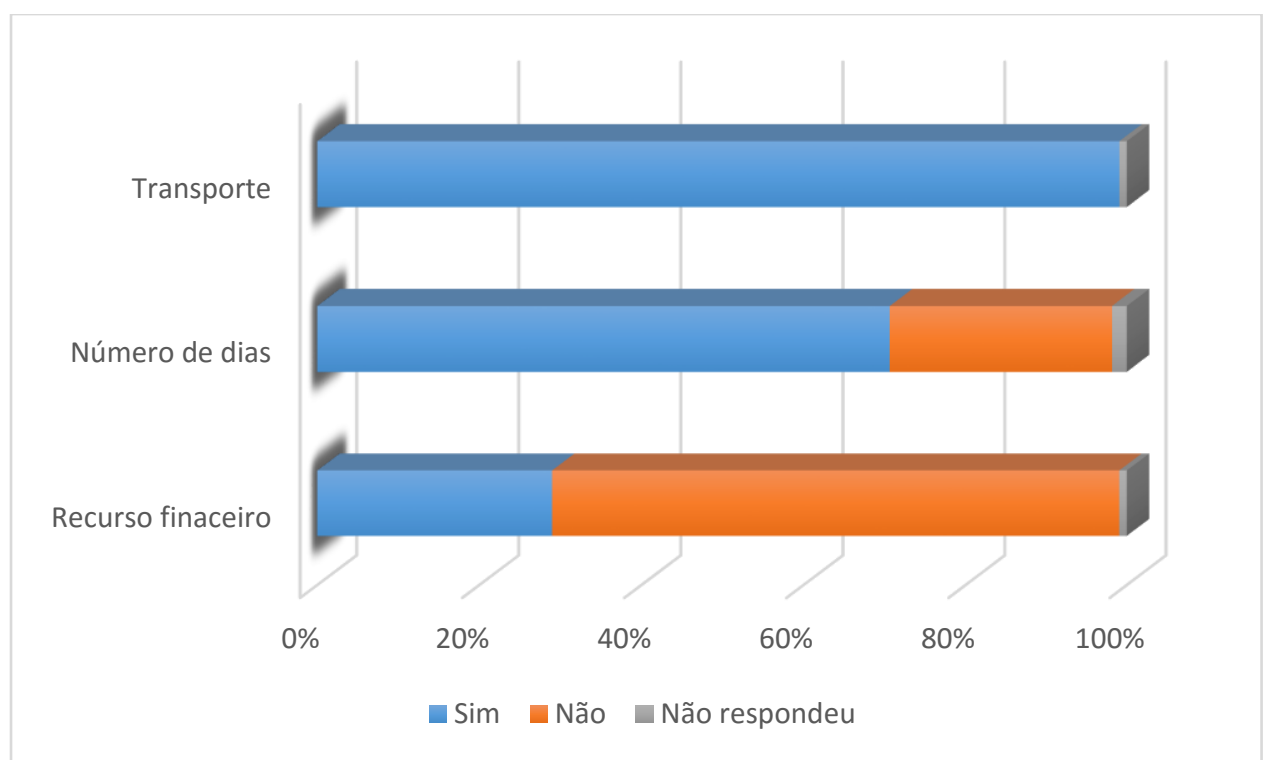

Gráfico 2. Satisfação em relação ao transporte utilizado, número de dias em atividade e recurso financeiro recebido. 


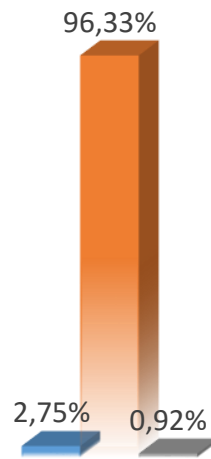

ATENDIMENTO COM ACOLHIMENTO

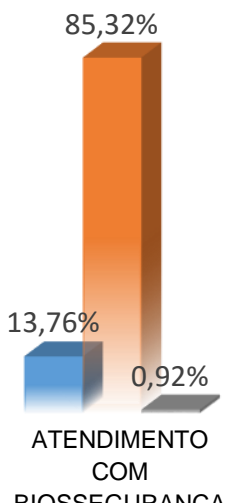

BIOSSEGURANÇA
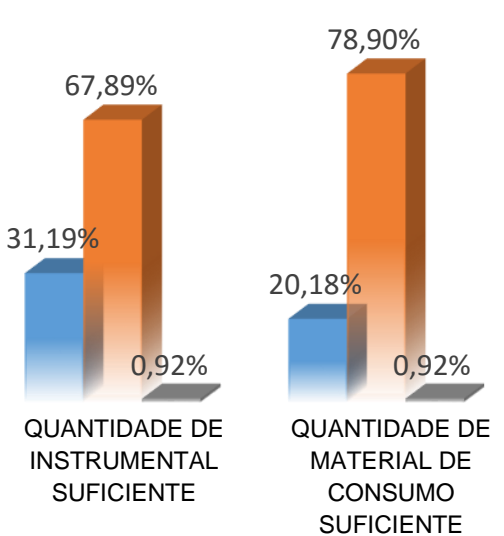

$8,26 \%$

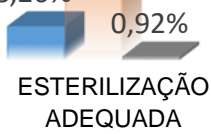

- Não Sim Não respondeu

Gráfico 3. Opinião dos estudantes em relação ao atendimento odontológico.

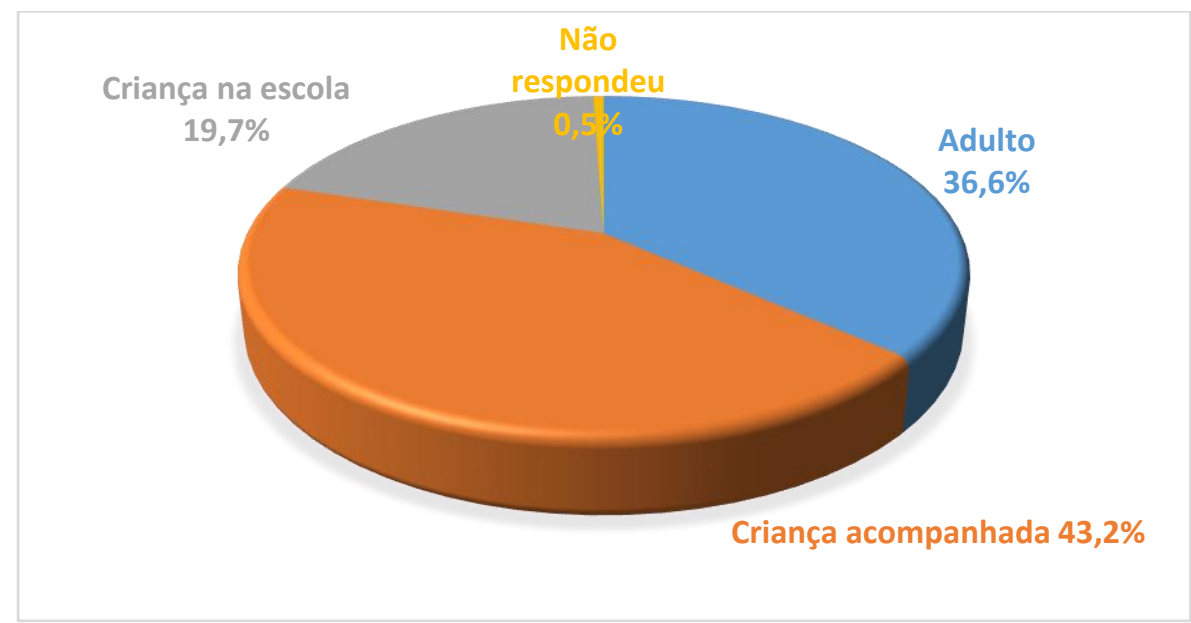

Gráfico 4. Situação em que o usuário se apresentou ao atendimento.

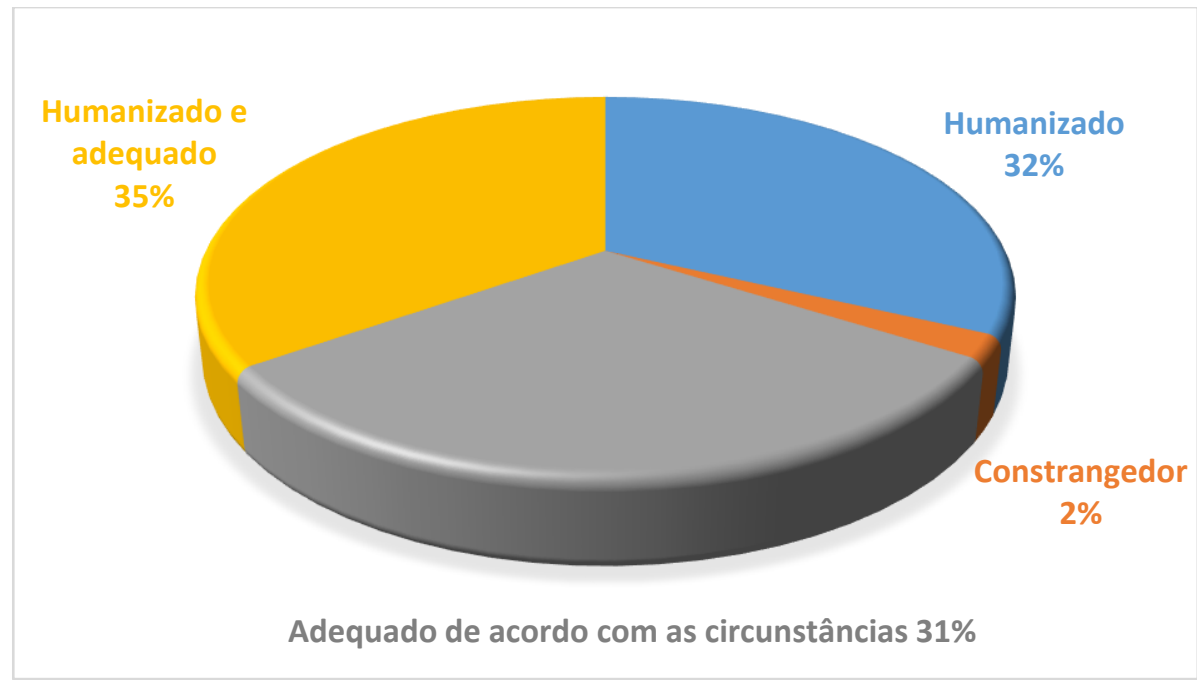

Gráfico 5. Percepção dos estudantes sobre o atendimento. 


\section{DISCUSSÃO}

Neste estudo realizado com alunos do Curso de Odontologia da UFPE que participaram da excursão didática, quase metade $(48,62 \%)$ teve a oportunidade de participar de mais de uma AE, tal resultado reflete a importância dessas experiências para a formação do cirurgião-dentista. Demonstrando essa importância, Moysés et al. $(2003)^{12}$ defendem que diversificar os cenários de ensino-aprendizagem favorece a integração do aluno à realidade social, às políticas sociais e ao SUS, com o objetivo de contextualizar a aprendizagem através da problematização com situações reais, estimulando o desenvolvimento de habilidades de negociação para decisões individuais e coletivas.

A participação em AE foi analisada por de Almeida Santa-Rosa et al $(2007)^{13}$, recebendo destaque um ponto que os alunos julgam importante na realização destas atividades, que é a possibilidade de aprenderem a trabalhar coletivamente nas comunidades, de entrarem em contato com realidades diversas e de fazerem um tipo de atendimento diferente do vivenciado nas práticas diárias intramuros. Ainda segundo os autores, ao exprimirem essas expectativas, os estudantes relataram que, até o oitavo período, a formação é voltada apenas para o atendimento individual do paciente e as atividades coletivas só são realizadas por estudantes que participam de projetos de extensão voltados para atividades educativas em saúde. Esse tipo de atividade vem proporcionar um trabalho em saúde coletiva mais concreto, difícil de ser vivenciado durante os períodos anteriores da graduação.

Dentre as habilidades desenvolvidas nas AE destaca-se também a capacidade de resolução de problemas relacionados à saúde bucal sem a utilização do arsenal tecnológico tão comum aos ambientes acadêmicos (cadeira odontológica, sistema de altarotação, etc) ${ }^{14}$. Neste contexto, os alunos foram indagados, no presente trabalho, a respeito da forma como os atendimentos foram realizados, além de questões como biossegurança, quantidade de instrumental e material de consumo e a logística empregada para realizar o ciclo de esterilização, onde a maioria das respostas foi positiva referindo como adequadas para o objetivo proposto.

Para de Araújo (2006) ${ }^{15}$ a grande falha do ensino odontológico é resultante da crença de que este não pode ser cientificamente embasado e socialmente científico ao mesmo tempo, estabelecendo-se um descompasso entre esses dois movimentos, sem possibilidade de harmonia.

Essa desarmonia é explicada pela filosofia cartesiana que influenciou as concepções de saúde e doença na medicina moderna, mantendo-se imperativa nos sistemas de saúde até hoje. A visão reducionista e mecanicista sobre o pensamento médico resultou no chamado modelo biomédico, que constitui as bases conceituais da moderna medicina científica. A medicina ocidental, assim como a odontologia e outras áreas afins, reconhece apenas as causas orgânicas da doença, excluindo as práticas alternativas ${ }^{16}$.

A modernização dos métodos não garante por si só que a universidade se integre ao seu meio, identifique-se com seus problemas e influa para mudar a realidade social. É necessário tratar, especificamente, da questão da mudança do conteúdo e das práticas. Estas não são decorrência automática de qualquer mudança metodológica, mas demandam uma transformação na cultura pedagógica da instituição, com um processo 
de capacitação em educação para docentes que, historicamente, eram "bons mestres" por serem "bons técnicos" 15 .

Por outro lado, a evolução tecnológica tem sido alvo de constante atenção e estudo, sendo tomadas medidas cada vez mais radicais na busca da superação de limites, o que reforça o caráter tecnicista do homem. Essa capacidade que o homem apresenta de criar e aperfeiçoar os serviços torna-o diferenciado desde os primórdios da humanidade ${ }^{17}$. Nessa perspectiva, pode-se considerar benéfica a evolução tecnológica visível na atualidade, no entanto, antigos conceitos de relacionamento e bem-estar estão sendo esquecidos nos ambientes de trabalho, e isso vem se refletindo no alto nível de insatisfação por parte dos usuários de serviços ${ }^{17}$. Neste sentido, a percepção dos alunos sobre a forma como ocorreu o relacionamento aluno/paciente/família e acolhimento foi avaliado como adequado e humanizado.

Sensibilizar os alunos frente a níveis socioeconômicos e culturais diferentes daqueles observados na capital e regiões metropolitanas faz parte do aprendizado. $\mathrm{O}$ estudo da relação entre indicadores de saúde e níveis socioeconômicos tem mostrado, em geral, uma relação diretamente proporcional, isto é, quanto pior o nível socioeconômico, mais comum em áreas rurais, pior os indicadores de saúde. Este postulado também se aplica para os indicadores de saúde bucal, especialmente para a cárie dentária ${ }^{18}$.

\section{CONCLUSÃO}

Através deste estudo foi possível concluir que as atividades extramuros desenvolvidas pelo curso de Odontologia da UFPE cumpriram seu principal objetivo que foi proporcionar aos alunos uma visão integral da realidade social e sanitária encontrada nas comunidades não assistidas pelos serviços básicos de saúde. Essa vivência da realidade contribui para a formação de um profissionalcidadão. Atividades extramurais como as excursões didáticas não podem ser entendidas como ações limitadas e isoladas, sendo importantes para a integralização do conhecimento.

\section{ABSTRACT \\ The community experiences during the tour of teaching in UFPE College of Dentistry - an extramural experience}

The aim of this study was to evaluate the perception of the students involved in the Tour Teaching project concerning the completeness of health care and integralization of learning, work and social practices. This is an analytical observational study. The sample included 109 students who participated in tours and belonging to the 7 th, 8 th 9 th or 10 th periods of the Dental course at Federal University of Pernambuco. The activities of the excursions were developed in the Pernambuco backlands cities. The questionnaire for collecting information from participants was composed of four sections: Personal information (name, age, gender and degree course period); Infrastructure required for the tour (transport, financial resources and duration of the activity); Clinical approach (reception, professional relationship with patients and families, biosafety and field of clinical activities); and Final results of the tour. The extramural activities in the communities served their main goal was to provide students with a comprehensive view of social reality and health found in communities not assisted by basic health services.

Descriptors: Teaching. Community Dentistry. Dental Education.

\section{REFERÊNCIAS}

1 Schmidt RAC. A questão ambiental na promoção da saúde: uma oportunidade 
de ação multiprofissional sobre doenças emergentes. Physis. 2007; 17 : 373-92.

Botazzo C, Manfredini MA, Narvai PC, Sao Pedro PF. Saúde bucal coletiva. Sao Paulo (Estado). Secretaria da Saude. Gepro de Saude Bucal, 1988. Sucupira AC, Mendes R. Promoção da Saúde: conceitos e definições. Sanare. 2003; 4: 7-10.

$4 \quad$ OMS. Carta de Ottawa. 1986.

5 Soares AN, Morgan BS, Santos FBO, Matozinhos FP, Penna CM de M. Crenças e práticas de saúde no cotidiano de usuários da rede básica de saúde. Rev enferm UERJ. 2014; 22: 838.

6 Mattos RA. Os sentidos da integralidade: algumas reflexões acerca de valores que merecem ser defendidos. In: Os sentidos da integralidade na atenção e no cuidado à saúde. ABRASCO. 2001. 43-68.

7 Júnior AM, Alves M, Nunes JP, Costa I do CC. Experiência extramural em hospital público e a promoção da saúde bucal coletiva. Rev Saúde Pública. 2005; 39:305-10.

8 Acioli S. A prática educativa como expressão do cuidado em Saúde Pública. Rev Bras Enferm. 2008; 61: 117-21.

9 MEC. Plano Nacional de Extensão Universitária. 2001.

10 Alves FBT, Gregol LRG, Czlusniak GD, Jiménez EEO. Ação integrada em Odontologia na comunidade: uma experiência da integração ensinoserviço. Rev Conexão UEPG. 2011; (2):230-9.
11

PROACAD/UFPE.

Excursões

Didáticas. 2013.

12 Moysés ST, Moysés SJ, Kriger L, Schmitt EJ. Humanizando a educação em Odontologia. Rev ABENO. 2003; 3: 58-64.

13 Almeida Santa-Rosa TT, Vargas AMD, Santa-Rosa TTA, Vargas AMD, Ferreira EF. O internato rural e a formação de estudantes do curso de Odontologia da UFMG. InterfaceComunic Saúde Educ. 2007; 11: 45166.

14 Brasil. Conselho Nacional de Secretários de Saúde. Atenção Primária e Promoção da Saúde. v. 8. 2007

15 Araujo ME. Palavras e silêncios na educação superior em odontologia. Ciênc Saúde Colet. 2006; 11: 179-82.

16 Siqueira CS, Lelis ÉR, Reis SM de ÁS. Saberes relevantes na formação do profissional da saúde. In: Universidade Federal de Uberlândia. 2008.

17 Lima ENA, Souza ECF. Percepção sobre ética e humanização na formação odontológica. RGO. 2010; 58: 231-8.

18 Tuon ACLF, Lacerda JT, Traebert J. Prevalência de cárie em escolares da zona rural de Jacinto Machado, SC, Brasil. Pesq Bra Odontoped e Clin Integ. 2007; 7: 277-84.

Correspondência para:

José Thadeu Pinheiro

e-mail: jtpendo@gmail.com

Curso de Odontologia

Universidade Federal de Pernambuco

Av. Prof. Moraes Rego, 1235

50670-901, Recife/PE 